\title{
Intenciones futuras de participar en un pequeño evento deportivo sostenible: influencia de la motivación, afición y satisfacción de los participantes en carreras solidarias
}

\author{
Future intentions to participate in a small sustainable sports event: influence of \\ motivation, interest and satisfaction of the participants in solidarity competitions
}

\author{
Rómulo J. González García ${ }^{1}$, Carlos Pérez Campos ${ }^{1}$, Fernando García Pascual ${ }^{2}$ \\ 1 Campus Capacitas. Universidad Católica San Vicente Mártir. Valencia, España. \\ 2 Departamento de Educación Física y Deportiva. Universitat de València. España. \\ CORRESPONDENCIA: \\ Rómulo J. González García \\ rj.gonzalez@ucv.es \\ CÓMO CITAR EL ARTÍCULO: \\ González-García, R. J., Pérez-Campos, C., \& García-Pascual, F. \\ (2021). Intenciones futuras de participar en un pequeño evento \\ deportivo sostenible: influencia de la motivación, afición y satis- \\ facción de los participantes en carreras solidarias. Cultura, Cien- \\ cia y Deporte, 16(50), 661-670. http://dx.doi.org/ 10.12800/ccd. \\ v16i50.1585

Abstrac

Small-scale sustainable sporting events have limited impact on the community, but there can be great benefits from holding them. For this reason, the celebration of this type of event has increased in recent years, on multiple occasions with solidarity purposes. However, few studies have been found which analyze the assessments of the participants. Therefore, it is the objective of this study to analyze the evaluations in reference to the motivation to participate, the liking for the sport practice and their satisfaction with the celebration of the event with respect to the future intentions of participation in this one. The results indicate that satisfaction with the event is the main variable that predicts future intentions of participating in this type of event. The aim is to provide information to the organizers of this type of event in order to improve organizational aspects that promote sustainability.

Key words: small sports event, future intentions, sustainability.

nes futuras, sostenibilidad. 


\section{Introducción}

Los principales objetivos para hacer frente a los desafíos presentes y futuros en materia de sostenibilidad, a escala mundial, se enmarcan en una serie de objetivos adoptados por las Naciones Unidas a conseguir en 2030, buscando una conexión entre los tres niveles de desarrollo sostenible: económico, social y ambiental (UN, 2015). Dentro de estos, se destaca la necesidad de equilibrar el progreso económico y la protección del medio ambiente, pero también teniendo en cuenta los intereses sociales (Dempsey et al., 2011). Sin embargo, el último informe desarrollado en 2019 señala la necesidad de una transformación urgente e intencional de los sistemas socioeconómicos para asegurar el bienestar humano, la salud de la sociedad y un impacto ambiental limitado (Järvensivu et al., 2019).

La celebración de eventos deportivos sostenibles puede ser una forma de contribuir con estos objetivos (González-Serrano et al., 2020). En la actualidad, los eventos deportivos se caracterizan por tener diversos niveles, ya sean para atletas profesionales o para aficionados, lo que provoca una aparición constante de nuevas disciplinas deportivas, algo que es capaz de llegar a un mayor número de individuos que son partícipes, en diverso grado, de este tipo de acontecimiento ( $\mathrm{Pa}$ rra-Camacho, et al., 2016). A medida que la industria deportiva continúa en constante crecimiento, tanto académicos como organizadores de eventos deben esforzarse por considerar las experiencias de los consumidores de servicios deportivos, entendiendo al participante como un factor esencial en el servicio ofrecido (Hyun y Jordan, 2019), o el impacto inmediato que tiene la celebración de este tipo de acontecimientos en la calidad de vida de las personas que viven en la comunidad de acogida del evento (Angosto-Sánchez, et al., 2016; Taks, et al., 2015). Los impactos asociados a la celebración de este tipo de acontecimientos son de diversa índole, identificando impactos económicos, turísticos-comerciales, físicos-medioambientales, sociales-culturales-deportivos, psicológicos y políticos-administrativos en las localidades que los acogen (Sánchez-Sáez, 2019).

A la hora de definir categóricamente el tamaño del evento, frecuentemente se hace referencia a los recursos necesarios para la organización y acogida de los asistentes, tanto de participantes como de espectadores (Tzanelli, 2017). En ese sentido, autores como Gratton, et al., (2000) proponen una categorización de eventos deportivos identificando cuatro grandes tipos de eventos. Asimismo, otros autores, como Wilson (2006), destacan que los eventos deportivos pequeños deben constituirse como otro tipo más en esta clasifi- cación, destacando eventos que se celebran anualmente y cuya actividad económica es limitada en comparación con los grandes eventos.

En la actualidad, la gestión de eventos deportivos tiende a utilizar una gestión basada en la sostenibilidad y el cuidado del medio ambiente (González-Serrano, et al., 2020). A través de los eventos deportivos, las instituciones sociales, políticas y económicas de una región pueden promover un mejor rendimiento económico, evaluado dentro de un paradigma, el cual destaca por la importancia de las interacciones sociales complejas y la sostenibilidad (Jones, 2005).

En general, los grandes eventos tienden a atraer niveles más elevados de apoyo empresarial y gubernamental debido a su alto perfil y repercusión, por lo que estos requieren de un mayor número de recursos (Preuss, 2009). Sin embargo, Gibson et al., (2003) señalan que es necesario conceptualizar los eventos de pequeña escala en términos relativos, ya que las definiciones indicadas anteriormente se aplican igualmente a las competiciones deportivas con apoyo popular local y, al mismo tiempo, a los acontecimientos deportivos que atraen a espectadores y participantes a nivel nacional e, incluso, a nivel internacional. La distinción entre los eventos de menor tamaño y los mega-eventos no solo hace referencia al tamaño del acontecimiento, sino también a la necesidad de aportar menos recursos públicos (ParraCamacho, et al., 2014). En ese sentido, los eventos deportivos de pequeña escala han tenido una alta incidencia en los últimos años (McKercher, 2016). Por su parte, Gibson et al. (2012), determinan estos como eventos que generalmente tienen un impacto limitado y un número de participantes que, a su vez, es mayor que el número de espectadores. Aunque los eventos más pequeños pueden generar una actividad económica limitada, sus resultados y beneficios netos para la comunidad local pueden ser positivos, lo que permite impulsar la economía local (Alguacil, et al., 2016; Kaplanidou y Gibson, 2010, Zhou y Kaplanidou, 2018). Sin embargo, el desarrollo de nuevos eventos y estimular la demanda turística de los eventos existentes requieren una mayor colaboración. Además, los criterios de decisión en este tipo de eventos pequeños son normalmente coherentes con las medidas para el desarrollo de modelos sostenible, incluyendo los impactos económicos, sociales, culturales y ambientales (Fotiadis, et al., 2016).

Escasas investigaciones han examinado en detalle los eventos a pequeña escala (Fotiadis, et al., 2016; Scholtz, 2019). Asimismo, estas se han centrado en la importancia de las actitudes hacia el evento y cómo la satisfacción con este resulta vital en la concepción de actitudes positivas, siendo un fuerte predictor de futuras intenciones de participación (Kaplanidou y 
Gibson, 2010). Por otra parte, se ha indagado en cómo el rendimiento deportivo de los participantes resulta estar relacionado con la satisfacción y, por tanto, este también afecta a las intenciones futuras de participación en el evento. Otros aspectos, como la motivación o la afición que los participantes tienen por realizar este tipo de actividad, podrían infundir la participación futura en el evento (Malchrowicz-Mosko y Poczta, 2018). De manera similar, se ha profundizado en el valor social que se obtiene por la participación en eventos, aportando beneficios sociales a la comunidad como la cohesión social, sentimientos de orgullo y apego a la comunidad (Gibson et al., 2012; GonzálezGarcía, et al., 2016; Zhou y Kaplanidou, 2018), aumentando también la conexión de la población local con el evento (Taks, 2013).

En concreto, el running se ha impulsado en las últimas décadas como una manera eficaz de lograr una buena forma física y promover el ejercicio físico a largo plazo (Rothschild, 2012). Relativo a este fenómeno, se han impulsado los eventos que acogen a este tipo de deportistas, a menudo con orientación inclusiva, abiertos a todos los públicos, cuyos participantes son en su mayoría corredores amateurs (Larsen y Bærenholdt, 2019). La literatura sobre este tipo de eventos se ha centrado principalmente en grandes eventos, como la maratón de Berlín (Edensor y Larsen, 2018) o la maratón de Londres (Shipway y Jones, 2008).

Los eventos de índole solidaria son una herramienta de recaudación de fondos puesto que los eventos deportivos generalmente populares, y las actividades que se realizan están vinculadas a un estilo de vida saludable (Hendriks y Peelen, 2013; Won, et al., 2010). Estos a menudo atraen a un mayor número de participantes cuya motivación principal es colaborar con la labor solidaria, sin necesidad de que la competición sea la principal motivación para ser partícipe en el evento (Hendriks y Peelen, 2013).

En línea a lo expuesto anteriormente, el deporte contribuye con la mejora de la salud de las personas (desarrollo social), a la vez que crea puestos de trabajo (desarrollo económico). Además, si se realiza teniendo en cuenta el impacto en el medio, puede ser una estrategia para el desarrollo sostenible de las poblaciones. En esta línea, Higham (1999), señala que los eventos deportivos a pequeña escala pueden ser una forma sostenible de desarrollo turístico para las comunidades.

\section{Motivación}

Los motivos para participar en eventos deportivos de pequeña escala destacan por ser una variable compleja (Larsen y Bærenholdt, 2019). MacLean y Hamm
(2007) describen la motivación como las razones, propósitos y objetivos que subyacen y perpetúan una acción individual. Diversos autores han estudiado las motivaciones para participar en una actividad deportiva organizada (Martinez-Caro y Martinez-García, 2007), señalando que estas están directamente relacionadas con los motivos de socialización, presentando con su participación una oportunidad para interactuar con diferentes personas (Zhou y Kaplanidou, 2018).

Tal y como señalaron Ko y Pastore (2004), es importante identificar los motivos de participación de los individuos, puesto que esto no solo determina el nivel de calidad del servicio, sino también la satisfacción que se pueda obtener de la participación en el evento. Concretamente, en eventos de índole solidaria facilita enormemente la relación entre organizadores y participantes, puesto que no existe una interacción negociocliente, sino que ambos colaboran en la labor (Larsen y Bærenholdt, 2019, Pérez-Campos y Alguacil, 2017).

\section{Satisfacción}

La satisfacción puede ser una variable importante que potencie las actitudes de los individuos (Oliver, 1980). La satisfacción se define como "una respuesta tanto evaluativa como emocional a un encuentro de servicio" (Cronin et al., 200. p. 204). Asimismo, esta variable puede orientar pautas de comportamiento en los consumidores de un servicio, incluyendo aquellos de índole deportiva (Kaplanidou y Gibson, 2010; Petrick, 2004). Por tanto, la satisfacción influye en las actitudes e intenciones de comportamiento referente a la participación, lo que puede indicar un importante factor a tener en cuenta a la hora de diseñar los servicios deportivos ofertados, incluido el evento deportivo. (Kaplanidou y Gibson, 2010). En ese sentido, diversos autores identifican variables como el rendimiento de los participantes o la calidad del servicio prestado, así como la consecución de metas como determinantes en la satisfacción (Angosto-Sánchez, et al., 2016; Du, et al., 2015; Hyun y Jordan, 2019).

\section{Afición}

Frecuentemente, los eventos de tipo solidario presentan una naturaleza más recreativa que competitiva, destacando el elemento de ocio en la participación de este tipo de eventos (Zhou y Kaplanidou, 2018). Por ese motivo, la participación en eventos diferencia a aquellos participantes cuya finalidad es la competición de los que únicamente pretenden participar como actividad de ocio (Getz y McConell, 2014). Se 
entiende como afición la actividad que se realiza meramente por placer, y el propósito de participación de los usuarios se centra la celebración del evento en sí mismo (Hyun y Jordan, 2019). No obstante, la afición por realizar actividad física no repercute en la participación en eventos de estas características de manera única (Funk, 2017).

\section{Intenciones futuras}

Diversos autores han tratado de analizar las variables relacionadas a las intenciones futuras de participación en eventos deportivos, independientemente del tamaño de estos. En ese sentido, Hyun y Jordan (2019) señalan que la intención de volver a participar está directamente relacionada con el éxito obtenido en el evento. Asimismo, las intenciones futuras también son susceptibles de modificarse por las expectativas previas que tenga el participante (Shonk y Chelladurai, 2008). A pesar de ello, no se puede afirmar que la celebración del evento deportivo sirva como valor principal a la hora de repetir participación en él; esta puede deberse a la finalidad con la que se organiza el evento, a la participación en el evento o a ambos casos (Shonk y Chelladurai, 2008). Es importante tener en cuenta las diferentes características particulares del consumidor a la hora de poder predecir la satisfacción de este, puesto que esto es un fuerte indicador de intención futura de consumo de dicho producto deportivo (Funk 2017; Hyun y Jordan, 2019).

En consecuencia, el objetivo de este estudio es analizar la relación existente entre la motivación para correr, el grado de afición que tienen los participantes por realizar esta actividad, la satisfacción con el evento y cuáles de estas variables resultan ser las mejores predictoras de las intenciones futuras de participar en un evento solidario de trail running.

\section{Método}

\section{Evento}

Esta investigación se ha llevado a cabo en la prueba denominada Goro Trail 2018, cuya celebración corresponde a la $2^{\text {a }}$ edición. Esta prueba de montaña, celebrada en el mes de julio de 2018, cuenta con alrededor de 400 corredores, que se distribuyen en tres modalidades diferentes, $6 \mathrm{~km}, 12 \mathrm{~km}$ y $21 \mathrm{~km}$. La celebración de este evento es de carácter benéfico, y los beneficios obtenidos de las inscripciones se destinan a diferentes colectivos en situación de vulnerabilidad y a entidades sin ánimo de lucro. El evento se celebró en el barrio de El Goro, cuya población asciende a 1427 habitantes (2018). Esta localidad pertenece al municipio de Telde, situado en la costa este de la isla de Gran Canaria (España).

\section{Participantes}

En esta investigación participaron un total de 100 corredores con edades comprendidas entre los 21 y los 56 años. La edad media ha sido de 39.21 años (DT=7.73), siendo la distribución por sexo de un $64 \%$ de hombres y un $36 \%$ mujeres.

\section{Instrumento}

El cuestionario estaba compuesto por un total de 26 ítems adaptados de otros estudios previamente validados (Prieto-Andreu, 2017) y evaluados por una escala de tipo Likert de 5 puntos. La fiabilidad se determinó mediante el alfa de Cronbach $(\alpha)$, la Fiabilidad Compuesta (FC) y la Varianza Media Extraída (AVE). Se ha obtenido un coeficiente de 0,91 para la escala de satisfacción con el evento $(F C=0.96 ; \mathrm{AVE}=0.51), 0,80$ en la escala de motivación para correr $(\mathrm{FC}=0.87$; $\mathrm{AVE}=$ $0.48)$ y 0,80 en escala relacionada con la afición por correr $(\mathrm{FC}=0.79 ; \mathrm{AVE}=0.56)$, así como una puntuación de 0,86 en la escala que mide las intenciones futuras de participar el en evento $(\mathrm{FC}=0.78 ; \mathrm{AVE}=0.64)$. El cuestionario general elaborado para medir las valoraciones de los participantes en la carrera obtuvo una puntuación de alfa de Cronbach de 0,87.

\section{Procedimiento}

Los datos fueron recogidos en el mes de julio de 2018, dos semanas después de la celebración del evento, a través de una encuesta estructurada y autoadministrada mediante la plataforma online LimeSurvey, mediante un muestreo intencional o de conveniencia. Para esta labor se contó con la colaboración de la organización. Los análisis estadísticos se llevaron a cabo mediante el paquete estadístico SPSS (Statistical Package for the Social Science, Versión 24).

Se han realizado un análisis descriptivo con el objetivo de conocer las medias y desviación estándar de los ítems correspondientes a las valoraciones de los participantes en el evento. La normalidad de los resultados fue examinada a través de los valores de asimetría y curtosis, todos ellos inferiores al criterio recomendando por Chou y Bentler (1995) de 3.0. También se ha realizado un análisis de correlación mediante el coeficiente de correlación de Pearson. Para evaluar la fiabilidad de la escala, se han tenido en cuenta medidas como el Alfa de Cronbach de cada uno de las variables 
analizadas (Hair et al., 1998) así como la FC y AVE. Con respecto al modelo de ecuaciones estructurales (SEM) se utilizó el programa EQS 6.2. Se utilizó una estimación por máximo de probabilidad (ML) con la corrección robusta de Satorra-Bentler (S-B X ${ }^{2}$ ) (Satorra y Bentler, 2001) a fin de controlar la posible incidencia de datos no normales. Se determinó la idoneidad del modelo a través del significado de Chi-cuadrado y su corrección robusta $\left(\chi^{2} ; S-B \chi^{2} ; \mathrm{p}>.01\right)$. Sin embargo, como esta estadística es susceptible al tamaño de la muestra, también se calcularon otros indicadores como la relación de $\chi^{2}$ y sus grados de libertad, y S-B X ${ }^{2}$ y sus grados de libertad, siendo aceptables los valores inferiores a cinco (Byrne, 2009). También se consideraron los índices de bondad de ajuste robusto de los modelos propuestos como el índice de ajuste comparativo (CFI) y el ajuste incremental (IFI), considerando indicadores de buenos valores de ajuste superiores a 0,90 (MacCallum y Austin, 2000). Por último, cuenta con el Root Mean-Square Error of Approximation (RMSEA), a partir del cual se pueden aceptar puntuaciones inferiores a 0,08 como indicador de un buen ajuste (Browne, y Cudeck, 1993).

\section{Resultados}

Tal y como se puede apreciar en la tabla 1 , las diferentes escalas asociadas a la valoración del evento tienen unos índices de fiabilidad aceptable. Asimismo,

Tabla 1. Análisis de las propiedades de los ítems de la escala valoración de los corredores sobre el evento.

\begin{tabular}{|c|c|c|c|c|c|}
\hline Cuestionario completo $(\alpha=.87)$ & Media (DT) & rjx & $\alpha-x$ & A & $\mathrm{C}$ \\
\hline \multicolumn{6}{|l|}{ Motivación $\alpha=.80$} \\
\hline Divertirme durante la competición & $4.60(.68)$ & .42 & .79 & -.1 .83 & 3.32 \\
\hline Sentirme atraído por el deporte & $4.13(.91)$ & .57 & .77 & -.68 & -.54 \\
\hline Encontrarme con otros corredores & $3.75(1.04)$ & .59 & .76 & -.44 & -.53 \\
\hline El atractivo que tienen las competiciones & $3.74(1.01)$ & .59 & .76 & -.47 & -.08 \\
\hline Sentirme parte del grupo de corredores & $3.49(1.16)$ & .55 & .77 & -.13 & -.88 \\
\hline Vencer a otros compañeros & $1.69(1.02)$ & .54 & .77 & 1.53 & 1,59 \\
\hline Deseo ser seleccionado y representar a mi país & $1.59(1.04)$ & .42 & .79 & 2.03 & 3.68 \\
\hline Obtener mejor tiempo que otro compañero & $1.55(.93)$ & .43 & .79 & 2.04 & 4.34 \\
\hline Total & $3.15(.62)$ & & & .65 & .21 \\
\hline \multicolumn{6}{|l|}{ Satisfacción con el evento $\alpha=.91$} \\
\hline Cumplimiento de horarios y puntualidad de las salidas & $4.23(.96)$ & .62 & .90 & -1.52 & 2.42 \\
\hline Servicio de guardarropa & $4.40(.86)$ & .71 & .89 & -1.65 & 3.16 \\
\hline Comunicación e información antes, durante y al finalizar la prueba & $4.42(.93)$ & .73 & .89 & -2.23 & 5.45 \\
\hline $\begin{array}{l}\text { Atención al corredor (avituallamiento líquido, servicio médico, servicio de masajes, servicio } \\
\text { de asesoramiento...) }\end{array}$ & $4.69(.72)$ & .81 & .89 & -3.42 & 13.97 \\
\hline $\begin{array}{l}\text { Obsequios y premios en función de la participación (prendas deportivas, premios, diploma } \\
\text { conmemorativo...) }\end{array}$ & $4,21(.99)$ & .57 & .90 & -1.31 & 1.22 \\
\hline Distancia actual de las carreras & $4.37(1.05)$ & .65 & .90 & -1.97 & 3.44 \\
\hline Diseño de los recorridos de las pruebas & $4.43(.88)$ & .67 & .89 & -2.06 & 5.10 \\
\hline $\begin{array}{l}\text { Utilización de las redes sociales para informar y comunicar noticias sobre el circuito de } \\
\text { carreras }\end{array}$ & $4,44(.88)$ & .70 & .89 & -2.08 & 5.17 \\
\hline Respecto a la organización de la Goro Trail 2018 & $4.67(.77)$ & .78 & .89 & -3.48 & 14.02 \\
\hline Total & $4.42(.68)$ & & & -2.79 & 11.40 \\
\hline \multicolumn{6}{|l|}{ Afición por correr $\alpha=.80$} \\
\hline Estoy deseoso de correr & $3.75(1.08)$ & .55 & .78 & -.67 & -.06 \\
\hline Correr es de vital importancia para mí & $3.39(1.24)$ & .61 & .76 & -.42 & -.71 \\
\hline Mi vida es mucho más rica porque corro & $3.55(1.22)$ & .71 & .73 & -.65 & -.42 \\
\hline Correr es agradable & $4.23(.84)$ & .46 & .79 & -1.09 & 1.36 \\
\hline Reorganizaría o cambiaría mi horario para satisfacer mi necesidad de correr & $3.24(1.21)$ & .45 & .79 & -.44 & -.58 \\
\hline Correr es el punto culminante del día & $2.95(1.28)$ & .59 & .76 & .04 & -.92 \\
\hline Total & $2.69(.49)$ & & & .46 & 2.31 \\
\hline \multicolumn{6}{|l|}{ Intenciones futuras $\alpha=.86$} \\
\hline Estoy dispuesto/a a continuar asistiendo a la Goro Trail el año que viene & $4.68(.51)$ & .49 & .98 & -1.24 & .49 \\
\hline Recomendaré la participación en la Goro Trail a otras personas & $4.75(.57)$ & .85 & .66 & -3.51 & 17.84 \\
\hline Hablaré bien de la Goro Trail a otras personas si me preguntan & $4.75(.59)$ & .87 & .65 & -3.43 & 16.26 \\
\hline Total & $4,72(, 49)$ & & & $-2,13$ & 5.29 \\
\hline
\end{tabular}

Nota: $\boldsymbol{\alpha}=$ Alfa de Cronbach. Media (M), desviación típica (DT), correlación ítem-total (rjx), Alpha de Cronbach si se elimina el elemento ( $\alpha-x)$, asimetría (A) y curtosis (C). 
destaca la escala de satisfacción con el evento y de intenciones futuras de participación, cuyas valoraciones medias de los ítems se sitúan entre $4,72(\mathrm{DT}=, 49)$ y 4,42 (DT = ,68) puntos de valoración media respectivamente. Respecto a la escala de intenciones futuras de participación, destacan los ítems relacionados a la recomendación de la prueba, con una valoración media de 4,75(DT=,57). Respecto a la escala de satisfacción con el evento, destaca el ítem asociado a la atención del corredor, con una valoración media de 4,69 (DT= ,72). Asimismo, la escala de afición por correr obtiene una valoración media general de 2,69 (DT = ,49) sobre 5 puntos, siendo esta la escala con menor valoración del instrumento. De ella, destaca el ítem "correr es el punto culminante del día”, con una valoración media de 2,95 (DT=1,28). Además, respecto a la escala de motivación por participar en el evento, esta ha obtenido una valoración general de 3,15 (DT= .62) puntos. De esta escala, destaca el ítem relacionado con finalidad de divertirse durante la competición como el ítem con mayor valoración $(\mathrm{M}=4,60 ; \mathrm{DT}=, 68)$. También se puede destacar como el ítem relacionado con la obtención de un mejor tiempo que el compañero obtiene la valoración media más baja del instrumento $(\mathrm{M}=1,55$; $\mathrm{DT}=, 93)$.

Igualmente, se ha realizado un análisis de correlación bivariada, de la cual los resultados han sido extraídos mediante el coeficiente de correlación de Pearson. Se observa cómo la motivación está correlacionada significativamente con la satisfacción con el evento $(r=250 ; \mathrm{p}<0,05)$ y la afición por correr $(r=529 ; \mathrm{p}$ $<0,01)$. A su vez, la satisfacción con el evento también se correlaciona significativamente con la afición por correr $(r=228$; $\mathrm{p}<0,05)$. y las intenciones futuras de participar nuevamente en el evento $(r=478$; $\mathrm{p}<0,01)$. Asimismo, no se observó que existiera una correlación entre la motivación y las intenciones futuras de participar en el evento $(r=165 ; \mathrm{p}>0,05)$, como tampoco existió la relación entre la afición por correr y las propias intenciones futuras $(r=185 ; \mathrm{p}>0,05)$.

Tabla 2. Coeficientes de correlación.

\begin{tabular}{ccccc}
\hline & $\mathrm{MC}$ & $\mathrm{SE}$ & $\mathrm{AC}$ & $\mathrm{IF}$ \\
\hline $\mathrm{MC}$ & 1 & & & \\
$\mathrm{SE}$ & $.250 *$ & 1 & & \\
$\mathrm{AC}$ & $.529 * *$ & $.228^{*}$ & 1 & \\
$\mathrm{IF}$ & 0.165 & $.478^{* *}$ & 0.185 & 1 \\
\hline
\end{tabular}

Nota: ${ }^{*}=p<0.05 ;{ }^{* *}=p<0.01 ;{ }^{* * *}=p<0.001 . ; M C=$ Motivación para correr; $S E=$ Satisfacción con el evento; $\mathrm{AC}=$ Afición por Correr; IF= Intenciones Futuras.

Los resultados obtenidos $\chi^{2}(\mathrm{df})=522.95$ (153) $\mathrm{p}<.001 ; \mathrm{S}-\mathrm{B} \chi^{2}(\mathrm{df})=170.618$ (132) $\mathrm{p}<.001, \mathrm{CFI}=.90$,
IFI=.91, RMSEA=.054) sugieren un ajuste adecuado del modelo, que explica el $49 \%$ de la varianza de las intenciones futuras ( $\mathrm{R} 2=.262, \mathrm{p}<0.01$ ). Se observa que, en el análisis de cada una de las variables predictoras, es la variable SE la única predictora $(\beta=.50$; $\mathrm{p}<.05)$. Por lo que respecta al resto de variables, $M C$ $(\beta=.08 ; p>.05)$, y $A C(\beta=-.07 ; p>.05)$, estas no resultaron tener relación estadísticamente significativa con la variable dependiente IF.

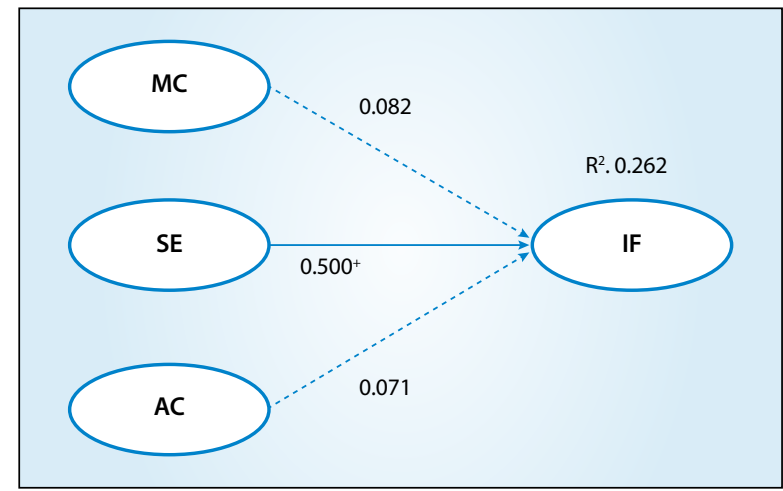

Figura 1. Modelo de ecuaciones estructurales. Nota. * $\mathrm{p} \leq .05 ; \mathrm{X}^{2}(\mathrm{df})=522.95$ (153) $\mathrm{p}<.001 ; \mathrm{S}-\mathrm{B} \mathrm{X}^{2}(\mathrm{df})=170.618$ (132) $\mathrm{p}<.001 ; \mathrm{CFI}=.90, \mathrm{IFI}=.91$, RMSEA $=.054$.

En la misma línea que en los análisis factoriales previos, dada la ausencia de normalidad multivariante, se utilizó el método Robusto de Estimación de Máxima Verosimilitud (MLR) con la finalidad de corregir dicha falta de normalidad. Como se puede observar en la tabla 3, los resultados del modelo de relaciones causales mostraron un buen ajuste global del mismo.

Tabla 3. Índices de bondad de ajuste del SEM sobre las percepciones de los residentes sobre los impactos asociados al turismo deportivo y el apoyo a su desarrollo en la comunidad.

\begin{tabular}{lc}
\hline Modelo & \\
\hline S-BX2(gl) & $170.618(123)$ \\
X2 (df) & $522.95(153)$ \\
X2 /df & 3.42 \\
RMSEA (IC) & $0.054(0.03-0.08)$ \\
CFI & 0,90 \\
IFI & 0,91 \\
\hline
\end{tabular}

Nota. S-B=Satorra Bentler; gl=grados de libertad; RMSEA = Error de Aproximación Cuadrático Medio ( $\leq .080)$; IC=Intervalo de Confianza RMSEA= error de aproximación cuadrático medio; $\mathrm{CFI}=$ Índice de Ajuste Comparado; IFI= Índice de Ajuste Incremental. CFI, IFI ( $\geq .90) ;$ X2/ gl ( $\leq 5.00)$.

En la tabla 4 se exponen todos los coeficientes estandarizados y su probabilidad asociada, así como, las relaciones planteadas. El modelo de relaciones causales presenta una $\mathrm{R}^{2}=.262$ que explica un $26.2 \%$ de la varianza. 
Tabla 4 . Resultados del modelo de relaciones causales con los coeficientes estandarizados y los valores críticos.

\begin{tabular}{ccc}
\hline Variables & Beta & t-valor \\
\hline MC $>$ IF & 0.082 & 1.08 \\
SE $>$ IF & 0.500 & $2.05^{*}$ \\
AC $>$ IF & 0.071 & 0.49 \\
\hline
\end{tabular}

Nota. ${ }^{*} p \leq, 05 . ; R 2=.262$

\section{Discusión}

El estudio de la participación en eventos deportivos, más concretamente en eventos de pequeña escala, ha suscitado el interés de los investigadores en la última década. Aunque recientemente se ha prestado atención al desarrollo de eventos deportivos sostenibles, estos se han centrado en eventos deportivos de gran escala (González-Serrano et al., 2020). Por lo tanto, existe un hueco en la literatura en lo que respecta al desarrollo sostenible de pequeños eventos deportivos En ese sentido, las variables que identifican las valoraciones presentadas por los participantes en el evento han sido analizadas desde una perspectiva multidimensional para intentar corregir esa laguna existente en la literatura. En consonancia con el estudio realizado, diferentes autores señalan cómo la satisfacción con el evento puede ser una de las variables fundamentales para comprender las intenciones de volver a asistir al evento. Por tanto, entender qué aspectos influyen en esa satisfacción puede ser importante a la hora de proveer e implementar servicios acordes a los intereses de los participantes (Kaplanidou y Gibson, 2010). De esta manera, conocer las variables que predicen las intenciones futuras de participar en eventos de pequeña escala puede ayudar a fomentar el desarrollo sostenible de las pequeñas poblaciones donde estas se celebran, ya que contribuye con la creación de hábitos físico-deportivos en dicha población, a la vez que supone una fuente de ingresos para esta. Sin embargo, siempre debe considerarse el impacto medioambiental, como es el caso de este pequeño evento objeto de estudio, el cual se desarrolló en un ambiente próximo a la localidad, y se tomaron ciertas medidas, como la utilización de material reutilizable.

En consonancia con los resultados obtenidos, trabajos como los de Chi y Qu (2008) y Kaplanidou y Gibson (2010) destacan que la satisfacción con el evento presenta una relación directa y significativa con las intenciones de volver a participar, incluyendo la voluntad de recomendar el evento como uno de los factores claves en esa intención. Otros trabajos, como los de Hendriks y Peelen (2013), señalan que la satisfacción con el evento es un factor que influye significativa- mente en la intención futura de regresar, hecho que también está en consonancia con el estudio de Bigne et al. (2001), que ponen de manifiesto que, aunque la satisfacción predice las intenciones de recomendar, esta no está claramente relacionada con la intención de volver a ser partícipe. En su estudio, Bigne et al., (2001) señalan que la satisfacción implica que el nivel de expectativas de los individuos se haya cumplido e incluso superado. En ese caso puede que las valoraciones de satisfacción de los encuestados reporten altos niveles de satisfacción. Este hecho puede explicar las valoraciones elevadas obtenidas en la carrera analiza$\mathrm{da}$, puesto que, al no estar consolidada en el calendario de eventos deportivos de la isla, muchos de los participantes obtuvieran unas perspectivas no lo suficientemente elevadas, por lo que resulta relativamente más sencillo obtener una buena impresión y, en consecuencia, una elevada satisfacción con el evento.

Sin embargo, trabajos como los de Kaplanidou y Vogt (2007) señalan que, concretamente en el evento de ciclismo analizado, la satisfacción no predijo las intenciones de volver a participar, contrariamente a los resultados obtenidos en esta investigación. Este hecho debe ser tomado con cautela debido a la naturaleza de los eventos analizados, en el caso de la Goro Trail se erige como una carrera solidaria, por lo que el carácter de competición no está presente, siendo el carácter solidario de la prueba una variable que influye en la satisfacción de los participantes.

La motivación de los corredores es uno de los aspectos más estudiados en el área de los eventos deportivos, señalando de esta manera la multidimensionalidad que influye en la misma. Aunque en este trabajo se ha aplicado un análisis de relación entre la motivación y las intenciones futuras, obteniendo que estas no presentan una relación estadísticamente significativa, diversos estudios señalan que la motivación podría tener incidencia en la satisfacción del evento (Zhou y Kaplanidou, 2018). Asimismo, se analizó la afición por correr como predictor de participación futura en el evento, observando que esta no resultaba ser estadísticamente significativa. Frecuentemente, la participación en eventos deportivos pequeños es importante en corredores amateurs, los cuales pretenden participar como actividad de ocio en estos acontecimientos (Getz y McConell, 2014); en ese sentido, no se puede afirmar que ser participe en este tipo de actividades de ocio puede ser indicador de intenciones futuras de participar en el evento.

Por lo tanto, los resultados de este estudio aportan información para los organizadores de eventos deportivos de pequeña escala, si quieren contribuir con el desarrollo sostenible de estos. Desarrollar estrategias 
que incrementen la satisfacción de los participantes debe ser una estrategia prioritaria para ellos. La responsabilidad de llevar a cabo políticas nacionales para una economía sostenible y un desarrollo humano sostenible depende de cada país (Leal Filho et al., 2019), así como de cada región. En este caso, los organizadores de eventos de pequeña escala también tienen un papel importante para ello. El impacto tanto económico como social y medioambiental positivo que puede generar los eventos deportivos de pequeña escala en estas localidades no debe infravalorarse. Además, debe tenerse en cuenta que siempre dependerá de la buena gestión de los organizadores de eventos deportivos, siendo la satisfacción de los participantes su principal indicador.

Finalmente, como limitaciones de este estudio, cabe destacar que los análisis realizados no son lo suficientemente consistentes como para determinar conclusiones generalizables a todos los eventos de esta naturaleza, por lo que existe la necesidad de seguir indagando en este sentido. También deberían haberse estudiado las valoraciones previas a la celebración del evento para identificar si realmente la valoración del evento tiene relación con las expectativas que poseen los participantes del mismo.

Por lo tanto, atendiendo a futuras líneas de investigación sería interesante analizar las variables de manera más extensa, tratando de identificar deferentes dimensiones que influyan en las variables principales del análisis. En ese sentido, se debe profundizar en el análisis de la satisfacción, indicando la implicación que se tiene en participar en un evento solidario, por si las características particulares del evento, concretamente la finalidad solidaria del mismo, pudiera ser una variable a observar en relación con la satisfacción de participar en el evento. Asimismo, sería interesante analizar la influen- cia de los diferentes aspectos sociodemográficos de los corredores. También, sería interesante realizar este tipo de estudios de manera longitudinal, observando si las valoraciones de los corredores varían en función de las modificaciones que se realicen en el evento.

\section{Conclusiones}

A modo de conclusión, es importante para los organizadores de pequeños eventos conocer las valoraciones y percepciones de los participantes con el objetivo de prestar un servicio de calidad para los asistentes. Independientemente de la implicación de estos en el evento, se debe prestar atención a la importancia de que los actos se realicen de manera sostenible, optimizando así la interacción de los participantes con el entorno. Concretamente, en este estudio se puede apreciar cómo la satisfacción con el evento destaca como factor predictor de las intenciones futuras de participación en la prueba. El resto de variables analizadas no presentaba una relación directa con las intenciones de participar en ediciones futuras del evento. Por lo tanto, es esencial garantizar unos servicios adecuados que certifiquen la satisfacción de los participantes, más aún en acontecimientos donde, dada la dimensión de los mismos, estos sean el agente mayoritario.

Estos datos son de especial relevancia para las personas encargadas de la gestión y organización de eventos deportivos. Con ello se adquiere información notable para la mejora de la oferta en este tipo de eventos con la finalidad de mantener a los participantes de estos satisfechos con su participación en la prueba, lo que implicará en su futura colaboración en este tipo de acontecimientos y su perpetuidad con el paso del tiempo. 


\section{BIBLIOGRAFÍA}

Alguacil, M., Pérez-Campos, C., Berenguer, S. A., \& Boquera, J. (2016). ¿La celebración de un evento promociona la ciudad como destino turístico entre los deportistas?. SPORT TK-Revista EuroAmericana de Ciencias del Deporte, 5, $123-136$

Angosto-Sánchez, S., López-Gullón, J. M., \& Díaz-Suárez, A. (2016). A Scale for Assessing Participants' Perceived Quality in Popular Sporting Events (CAPPEP V2. 0). Journal of Sports Economics \& Management, 6(2), 69-84

Angosto-Sánchez, S., Morán-Navarro, R., Martínez-Cava, A., \& LópezGullón, J. M. (2016). Calidad percibida en una prueba de triatlón. SPORT TK-Revista EuroAmericana de Ciencias del Deporte, 5(1), 81-84

Bigne, J. E., Sanchez, M. I., \& Sanchez, J. (2001). Tourism image, evaluation variables and after purchase behaviour: inter-relationship. Tourism Management, 22(6), 607-616.

Browne, M., \& Cudeck, R. (1993). Alternative ways of assessing model fit. In: K. Bollen, and J. Long (Eds.) Testing Structural Equation Models, (pp. 136-162). Beverly Hills, CA: Sage

Byrne, B. M. (2009). Structural equation modeling with amos: Basic concepts, applications, and programing. (2nd ed.). New York, NY: Routledge.

Chi, C. G. Q., \& Qu, H. (2008). Examining the structural relationships of destination image, tourist satisfaction and destination loyalty: An integrated approach. Tourism Management, 29(4), 624-636.

Chou, C.-P., \& Bentler, P. M. (1995). Estimation and tests in structural equation mod- eling. In R. H. Hoyle (Ed.), Structural equation modeling: Concepts, issues, and ap-plications (pp. 37-55). Thousand Oaks, CA: Sage.

Cronin Jr, J. J., Brady, M. K., \& Hult, G. T. M. (2000). Assessing the effects of quality, value, and customer satisfaction on consumer behavioral intentions in service environments. Journal of Retailing, 76(2), 193-218.

Dempsey N, Bramley G, Power S,. \& Brown C. (2011). The social dimension of sustainable development: defining urban social sustainability. Sustainable Development, 19, 289-300.

Du, J., Jordan, J. S., \& Funk, D. C. (2015). Managing mass sport participation: Adding a personal performance perspective to remodel antecedents and consequences of participant sport event satisfaction. Journal of Sport Management, 29(6), 688-704.

Edensor, T., \& Larsen, J. (2018). Rhythmanalysing marathon running:'A drama of rhythms'. Environment and Planning A: Economy and Space, 50(3), 730-746

Fotiadis, A. K., Vassiliadis, C. A., \& Sotiriadis, M. D. (2016). The preferences of participants in small-scale sport events: A conjoint analysis case study from Taiwan. Turizam: međunarodni znanstveno-stručni časopis, 64(2), 175-187.

Fotiadis, A., Vassiliadis, C., \& Yeh, S. P. (2016). Participant's preferences for small-scale sporting events: A comparative analysis of a Greek and a Taiwanese cycling event. EuroMed Journal of Business, 11(2), 232247.

Funk, D.C. (2017). Introducing a sport experience design (SX) framework for sport consumer behaviour research. Sport Management Review, 20, 145-158.

Getz, D. \& McConnell, A. (2014). Comparing Trail Runners and Mountain Bikers: Motivation, Involvement, Portfolios, and Event-Tourist Careers. Journal of Convention \& Event Tourism, 15(1), 69-100.

Gibson, H. J., Kaplanidou, K., \& Kang, S. J. (2012). Small-scale event sport tourism: A case study in sustainable tourism. Sport Management Review, 15(2), 160-170.

Gibson, H. J., Willming, C., \& Holdnak, A. (2003). Small-scale event sport tourism: Fans as tourists. Tourism management, 24(2), 181-190.

González-García, R. J., Parra-Camacho, D., González-Serrano, M. H., \& Añó-Sanz, V. (2016). Sports events as enhancing tourism in Gran Canaria: an analysis from the social perception. Revista Intercontinental de Gestão Desportiva, 6(1), 108-120.

González-Serrano, M. H., Añó Sanz, V., \& González-García, R. J. (2020). Sustainable Sport Entrepreneurship and Innovation: A Bibliometric Analysis of This Emerging Field of Research. Sustainability, 12(12), 5209.
Gratton, C., Dobson, N., \& Shibli, S. (2000). The economic importance of major sports events: a case-study of six events. Managing leisure, 5(1), 17-28.

Hair, J. F., Anderson, R. E., Tatham, R. L., \& Black, W. C. (1998). Multivariate data analysis (5th edn).). New Jersey: Prentice Hall.

Hendriks, M., \& Peelen, E. (2013). Personas in action: linking event participation motivation to charitable giving and sports. International Journal of Nonprofit and Voluntary Sector Marketing, 18(1), 60-72.

Higham, J. (1999). Commentary-Sport as an avenue of tourism development: An analysis of the positive and negative impacts of sport tourism. Current Issues inTourism, 2(1), 82-90.

Hyun, M., \& Jordan, J. S. (2019). Athletic goal achievement: A critical antecedent of event satisfaction, re-participation intention, and future exercise intention in participant sport events. Sport Management Review. In Press.

Järvensivu, P., Toivanen, T., Vadén, T., Lähde, V., Majava, A. \& Eronen, J.T. (2018). Governance of economic transition: Invited background document for global sustainable development report 2019[Online]. Disponible en: http://bios.fi/bios-governance_of_economic_transition.pdf [consultado 17 febrero 2021]

Jones, C. (2005). Major events, networks and regional development. Regional studies, 39(2), 185-195

Kaplanidou, K., \& Gibson, H. J. (2010) Predicting Behavioral Intentions of Active Event Sport Tourists: The Case of a Small-scale Recurring Sports Event. Journal of Sport \& Tourism, 15:2, 163-179, DOI: 10.1080/14775085.2010.498261

Kaplanidou, K., \& Vogt, C. (2007). The interrelationship between sport event and destination image and sport tourists' behaviours. Journal of Sport \& Tourism, 12(3-4), 183-206.

Ko, Y. J., \& Pastore, D. L. (2004). Current issues and conceptualizations of service quality in the recreation sport industry. Sport marketing quarterly, 13(3), 158-166.

Larsen, J., \& Bærenholdt, J. O. (2019). Running together: The social capitals of a tourism running event. Annals of Tourism Research, 79, 102788.

Leal Filho, W., Tripathi, S. K., Andrade Guerra, J. B. S. O. D., Giné-Garriga, R., Orlovic Lovren, V., \& Willats, J. (2019). Using the sustainable development goals towards a better understanding of sustainability challenges. International Journal of Sustainable Development \& World Ecology, 26(2), 179-190.

MacCallum, R. C., \& Austin, J. T. (2000). Applications of structural equation modeling in psychological research. Annual Review of Psychology, 51, 201-226

MacLean, J., \& Hamm, S. (2007). Motivation, commitment, and intentions of volunteers at a large Canadian sporting event. Leisure, 31(2), 523-556.

Malchrowicz-Mośko, E., \& Poczta, J. (2018). A Small-Scale Event and a Big Impact: Is This Relationship Possible in the World of Sport? The Meaning of Heritage Sporting Events for Sustainable Development of Tourism-Experiences from Poland. Sustainability, 10(11), 4289.

Martinez-Caro, L., \& Martinez- Garcia, J. A. (2007). Measuring perceived service quality in urgent transport service. Journal of Retailing and Consumer Services, 14(1), 60-72.

McKercher, B. (2016). Towards a taxonomy of tourism products. Tourism Management, 54, 196-208.

Oliver, R. L. (1980). A cognitive model of the antecedents and consequences of satisfaction decisions. Journal of Marketing Research, 17(4), 460-469.

Parra-Camacho, D., Calabuig, F., Añó-Sanz, V., Ayora-Pérez, D. \& Núñez-Pomar, J. (2014). El impacto de un evento deportivo mediano: percepción de los residentes de la comunidad de acogida. Retos. Nuevas tendencias en Educación Física, Deporte y Recreación, (26), 88-93.

Parra-Camacho, D., González-García, R. J., Añó-Sanz, V., \& Ayora-Pérez, D. (2016). Percepción de los visitantes sobre el impacto social y sus intenciones con respecto a la celebración de un evento deportivo de pequeña escala. Revista de Psicología del Deporte, 25(1) 93-96.

Pérez-Campos, C., \& Alguacil, M. (2017). Validation of a scale to measure athletes' perception of sporting events service quality. Journal of Sports Economics \& Management, (7), 165-175. 
Petrick, J. F. (2004). The roles of quality, value, and satisfaction in predicting cruise passengers' behavioral intentions. Journal of Travel Research, 42(4), 397-407.

Preuss, H. (2009). Opportunity costs and efficiency of investments in mega sport events. Journal of Policy Research in Tourism, Leisure and Events, 1(2), 131-140.

Rothschild, C. E. (2012). Primitive running: a survey analysis of runners' interest, participation, and implementation. The Journal of Strength \& Conditioning Research, 26(8), 2021-2026.

Sánchez-Sáez, J. A. (2019). Los eventos deportivos como instrumento de desarrollo local. Cultura, Ciencia y Deporte, 14(41), 91-92.

Satorra, A., \& Bentler, P. M. (2001). A scaled difference chi-square test statistic for moment structure analysis. Psychometrika, 66, 507-514.

Scholtz, M. (2019). Does a small community (town) benefit from an international event?. Tourism Management Perspectives, 31, 310-322.

Shipway, R. \& Jones, I. (2008), "The Great Suburban Everest: an 'Insiders' perspective on experiences at the 2007 Flora London Marathon". Journal of Sport \& Tourism, 13(1), 61-77.

Shonk, D. J., \& Chelladurai, P. (2008). Service quality, satisfaction, and intent to return in event sport tourism. Journal of Sport Management, 22(5), 587-602.
Taks, M. (2013). Social sustainability of non-mega sport events in a global world1. European Journal for Sport and Society, 10(2), 121-141.

Taks, M., Chalip, L., \& Green, B. C. (2015). Impacts and strategic outcomes from non-mega sport events for local communities. European Sport Management Quarterly, 15(1), 1-6.

Tzanelli, R. (2017). Mega-events as economies of the imagination: Creating atmospheres for Rio 2016 and Tokyo 2020. Routledge.

UN (2015) Transforming our world: the 2030 agenda for sustainable development. Available athttp://www.un.org/pga/wp-content/uploads/ sites/3/2015/08/120815 outcome-document-of-Summit-foradoption-of-the-post-2015-development-agenda.pd

Wilson, R. (2006). The economic impact of local sport events: significant, limited or otherwise? A case study of four swimming events. Manag ing Leisure, 11(1), 57-70.

Won, D., Park, M., \& Turner, B. A. (2010). Motivations for participating in health related charity sport events. Journal of Venue and Event Management, 1(1), 17-44

Zhou, R., \& Kaplanidou, K. (2018). Building social capital from sport event participation: An exploration of the social impacts of participatory sport events on the community. Sport Management Review, 21(5), 491-503. 